Modern Physics Letters A

(C) World Scientific Publishing Company

\title{
SCATTERING IN NONCOMMUTATIVE QUANTUM MECHANICS
}

\author{
S. A. Alavi \\ Department of Physics, Tarbiat Moallem University of Sabzevar, \\ P.O.Box 397, Sabzevar, Iran \\ Sabzevar House of Physics, Javan-Sara, Asrar Avenue, Sabzevar, Iran. \\ alavi@sttu.ac.ir \\ alialavi@fastmail.us
}

Received (Day Month Year)

\begin{abstract}
We derive the correction due to noncommutativity of space on Born approximation, then the correction for the case of Yukawa potential is explicitly calculated. The correction depends on the angle of scattering. Using partial wave method it is shown that the conservation of the number of particles in elastic scattering is also valid in noncommutative spaces which means that the unitarity relation is held in noncommutative spaces. We also show that the noncommutativity of space has no effect on the optical theorem. Finally we study Gaussian function potential in noncommutative spaces which generates delta function potential as $\theta \rightarrow 0$.

Keywords: Noncommutative quantum mechanics, Born approximation, Partial wave method.

PACS Numbers: 03.65.-w, 02.20.a.
\end{abstract}

\section{Introduction}

Recently there have been notable studies on the formulation and possible experimental consequences of extensions of the standard (usual) quantum mechanics in the noncommutative spaces! 1 - 21] The study on noncommutative spaces is much important for understanding phenomena at short distances beyond the present test of QED.

In field theories the noncommutativity is introduced by replacing the standard product by the star product. For a manifold parameterized by the coordinates $x_{i}$, the noncommutative relations can be written as

$$
\left[\hat{x}_{i}, \hat{x}_{j}\right]=i \theta_{i j} \quad\left[\hat{x}_{i}, \hat{p}_{j}\right]=i \delta_{i j} \quad\left[\hat{p}_{i}, \hat{p}_{j}\right]=0,
$$

where $\theta_{i j}=\frac{1}{2} \epsilon_{i j k} \theta_{k}$. NCQM is formulated in the same way like the standard quantum mechanics SQM (quantum mechanics in commutative spaces), that is in terms of the same dynamical variables represented by operators in a Hilbert space and a state vector that evolves according to the Schroedinger equation

$$
i \frac{d}{d t}\left|\psi>=H_{n c}\right| \psi>,
$$


we have taken into account $\hbar=1 . H_{n c} \equiv H_{\theta}$ denotes the Hamiltonian for a given system in the noncommutative space. In the literatures two approaches have been considered for constructing the NCQM:

a) $H_{\theta}=H$, so that the only difference between SQM and NCQM is the presence of a nonzero $\theta$ in the commutator of the position operators i.e. Eq.(1).

b) By deriving the Hamiltonian from the Moyal analog of the standard Schroedinger equation:

$$
i \frac{\partial}{\partial t} \psi(x, t)=H\left(p=\frac{1}{i} \nabla, x\right) * \psi(x, t) \equiv H_{\theta} \psi(x, t),
$$

where $H(p, x)$ is the same Hamiltonian as in the standard theory, and as we observe the $\theta$ - dependence enters now through the star product! 19$]$ In [21], it is shown that these two approaches lead to the same physical theory.

It has been shown that many physical quantities, for example the spectrum of hydrogen atom, 1 Lamb shift 1 and Berry's phase, 17 receive corrections due to noncommutativity of space for which these corrections depend on the noncommutativity parameter. It is also shown that the noncommutativity of space has no effect on algebras of observables of systems of identical particles and on Pauli exclusion principle. 318

Scattering theory is of broad interest and importance to all branches of physics from particle to condensed matter physics. In this paper we study some important aspects of scattering theory in the framework of noncommutative quantum mechanics.

In SQM physicists usually use two methods to study scattering process:

a) Born approximation,

b) Partial wave method.

Let us start with the definition of star product:

$$
(f \star g)(x)=\left.\exp \left(\frac{i}{2} \theta_{\mu \nu} \partial_{x_{\mu}} \partial_{y_{\nu}}\right) f(x) g(y)\right|_{x=y} .
$$

Since the noncommutativity parameter, if it is non-zero, should be very small compared to the length scales of the system, one can consider the noncommutativity effects to the first order, then we have

$$
(f \star g)=f(x) g(x)+\frac{i}{2} \theta_{\mu \nu} \partial_{\mu} f \partial_{\nu} g+\mathcal{O}\left(\theta^{2}\right) .
$$

In what follows, we use this equation to study scattering in noncommutative quantum mechanics.

\section{Born approximation in noncommutative spaces}

The matrix elements $M_{f i}$ are defined as follows

$$
M_{f i}^{N C}=\left\langle\psi_{f}|V| \psi_{i}\right\rangle_{N C}=\int d^{3} r e^{-i \frac{\vec{p}_{f} \cdot \vec{r}}{\hbar}} \star V(\vec{r}) \star e^{i \frac{\vec{p}_{i} \cdot \vec{r}}{\hbar}} .
$$


Using the definition of star product to the first order the integrand can be expanded as

$$
\begin{aligned}
& e^{-i \frac{\vec{p}_{f} \cdot \vec{r}}{\hbar}} V(\vec{r}) e^{i \frac{\vec{p}_{i} \cdot \vec{r}}{\hbar}}+\frac{i}{2} e^{-i \frac{\vec{p}_{f} \cdot \vec{r}}{\hbar}} \theta_{\mu \nu}(\nabla V)_{\mu}\left(i \frac{\vec{p}_{\nu}^{i}}{\hbar}\right) e^{i \frac{\vec{p}_{i} \cdot \vec{r}}{\hbar}} \\
+ & \frac{i}{2} e^{-i \frac{\vec{p}_{f} \cdot \vec{r}}{\hbar}} \theta_{\mu \nu}(\nabla V)_{\nu}\left(-i \frac{\vec{p}_{\mu}^{f}}{\hbar}\right) e^{i \frac{\vec{p}_{i} \cdot \vec{r}}{\hbar}}+\frac{i}{2} \theta_{\mu \nu}\left(-i \frac{\vec{p}_{\mu}^{f}}{\hbar}\right)\left(i \frac{\vec{p}_{\nu}^{i}}{\hbar}\right) V(\vec{r}) e^{i \frac{\left(\vec{p}_{i}-\vec{p}_{f}\right) \cdot \vec{r}}{\hbar}} .
\end{aligned}
$$

Therefore we have

$$
\begin{aligned}
\left\langle\psi_{f}|V| \psi_{i}\right\rangle_{N C} & =\left\langle\psi_{f}|V| \psi_{i}\right\rangle_{C}+\int d^{3} r \frac{1}{2 \hbar} \theta_{\mu \nu}\left(p_{\mu}^{i}+p_{\mu}^{f}\right)(\nabla V)_{\nu} e^{i \frac{\left(\vec{p}_{i}-\vec{p}_{f}\right) \cdot \vec{r}}{\hbar}} \\
& +\int d^{3} r \frac{i}{2 \hbar^{2}} \theta_{\mu \nu} p_{\mu}^{f} p_{\nu}^{i} V(\vec{r}) e^{i \frac{\left(\vec{p}_{i}-\vec{p}_{f}\right) \cdot \vec{r}}{\hbar}}
\end{aligned}
$$

The first term is the Born approximation in commutative space, the second and the third terms show the effects of noncommutativity of space. Now let us derive these corrections, we have

$$
\frac{i}{2 \hbar^{2}} \theta_{\mu \nu} p_{\mu}^{f} p_{\nu}^{i}=\frac{i}{4 \hbar^{2}} \epsilon_{\mu \nu \sigma} p_{\mu}^{f} p_{\nu}^{i} \theta_{\sigma}=\frac{i}{4 \hbar^{2}}\left(\vec{p}_{f} \times \vec{p}_{i}\right) \cdot \vec{\theta} .
$$

If we take the component of $\vec{\theta}$ in the direction perpendicular to the plane of $\vec{p}_{f}$ and $\vec{p}_{i}$ (z-axis) equal to $\theta_{3}=\theta$ and put the rest $\theta$-components to zero(which can be done by a rotation or a redifinition of coordinates), then we have

$$
\frac{i}{4 \hbar^{2}}\left(\vec{p}_{f} \times \vec{p}_{i}\right) \cdot \vec{\theta}=\frac{i \theta}{4 \hbar^{2}} p^{2} \sin \phi,
$$

where $\phi$ is the angle between $\vec{p}_{f}$ and $\vec{p}_{i}$.

In the same way we can calculate the contribution of the third term in Eq.(8).

Then for Yukawa potential $V(r)=Z_{1} Z_{2} e^{2} \frac{e^{-\frac{r}{a}}}{r}$, the differential cross section in a noncommutative space is

$$
\left(\frac{d \sigma}{d \Omega}\right)_{N C}=\left(\frac{Z_{1} Z_{2} e^{2}}{4 E \sin ^{2}\left(\frac{\phi}{2}\right)+\frac{\hbar^{2}}{2 m a^{2}}}\right)^{2}\left[1+\frac{9 \theta^{2} p^{4}}{16 \hbar^{4}} \sin ^{2} \phi\right] .
$$

We observe that the correction depends on the angle of scattering $\phi$.

\section{Partial wave method in noncommutative spaces-Partial wave expansion of scattering amplitude}

We consider a sphere of radius $r=R$. Inside the sphere there may be given a potential $V(r)$; outside the sphere the potential may vanish. A beam of particles described by a plane wave is scattered at this potential field. We shall compute the 
scattering amplitude by expansion into a series of partial waves.

In the domain $r<R$ the wave function can be written as

$$
u^{\text {inside }}\left(r, \theta^{\prime}\right)=\sum_{\ell=0}^{\infty} i^{\ell}(2 \ell+1)\left[\frac{1}{k r} \chi_{\ell}(k r)\right] \star P_{\ell}\left(\cos \theta^{\prime}\right) .
$$

The boundary condition $\chi_{\ell}(0)=0$, ensures that $R_{\ell}(r)=\frac{\chi_{\ell}(r)}{r}$ be finite at $r=0$. Outside the sphere $r=R$ we write

$$
u^{\text {outside }}\left(r, \theta^{\prime}\right)=\sum_{\ell=0}^{\infty} i^{\ell}(2 \ell+1)\left\{\frac{1}{k r}\left[j_{\ell}(k r)+\frac{1}{2} \alpha_{\ell} h_{\ell}^{(1)}(k r)\right]\right\} \star P_{\ell}\left(\cos \theta^{\prime}\right) .
$$

Up to the first order in noncommutativity parameter $\theta$, we have

$$
\begin{aligned}
u^{\text {inside }}\left(r, \theta^{\prime}\right) & =\frac{1}{k} \sum_{\ell=0}^{\infty} i^{\ell}(2 \ell+1)\left\{\frac{1}{r} \chi_{\ell}(k r) P_{\ell}\left(\cos \theta^{\prime}\right)\right. \\
& \left.+\frac{i}{2} \theta_{\mu \nu} \partial_{\mu}\left(\frac{1}{r} \chi_{\ell}(r)\right) \partial_{\nu} P_{\ell}\left(\cos \theta^{\prime}\right)\right\}+\mathcal{O}\left(\theta^{2}\right)
\end{aligned}
$$

and

$$
\begin{aligned}
u^{\text {outside }}\left(r, \theta^{\prime}\right) & =\frac{1}{k} \sum_{\ell=0}^{\infty} i^{\ell}(2 \ell+1)\left\{\left[j_{\ell}(k r)+\frac{1}{2} \alpha_{\ell} h_{\ell}^{(1)}(k r)\right] P_{\ell}\left(\cos \theta^{\prime}\right)\right. \\
& \left.+\frac{i}{2} \theta_{\mu \nu} \partial_{\mu}\left[\frac{1}{r}\left(j_{\ell}(k r)+\frac{1}{2} \alpha_{\ell} h_{\ell}^{(1)}(k r)\right)\right] \partial_{\nu} P_{\ell}\left(\cos \theta^{\prime}\right)\right\}+\mathcal{O}\left(\theta^{2}\right) .
\end{aligned}
$$

One can express the coefficients $\alpha_{\ell}$ by the Logarithmic derivatives $L_{\ell}$

$$
L_{\ell}=\left(\frac{d \log \chi_{\ell}}{d \log r}\right)_{r=R} .
$$

To do this, we use the continuity of both $\chi_{\ell}$ and $\frac{d \chi_{\ell}}{d r}$ when passing through the sphere $r=R$ and divide the second by the first one, then we get

$$
\begin{aligned}
L_{\ell} & =x \frac{A+\frac{\theta}{2} B}{C+\frac{\theta}{2} D} \\
A & =\frac{1}{x}\left[j_{\ell}^{\prime}(x)+\frac{1}{2} \alpha_{\ell} h_{\ell}^{(1) \prime}(x)\right], \\
B & =\frac{1}{x^{2}} j_{\ell}^{\prime}(x)+\frac{1}{2 x^{2}} \alpha_{\ell} h_{\ell}^{(1) \prime}(x)-\frac{1}{x} j_{\ell}^{\prime \prime}(x)-\frac{1}{2 x} \alpha_{\ell} h_{\ell}^{(1) \prime \prime}(x), \\
C & =\frac{1}{x}\left[j_{\ell}(x)+\frac{1}{2} \alpha_{\ell} h_{\ell}^{(1)}(x)\right], \\
D & =-\frac{1}{x^{2}} j_{\ell}(x)-\frac{1}{2 x^{2}} \alpha_{\ell} h_{\ell}^{(1)}(x)+\frac{1}{x} j_{\ell}^{\prime}(x)+\frac{1}{2 x} \alpha_{\ell} h_{\ell}^{(1) \prime}(x),
\end{aligned}
$$

where $x=k R$, and the prime denotes differentiation with respect to the argument $k r$. Inversely we have

$$
\alpha_{\ell}=2 \frac{E+\frac{\theta}{2} F}{G+\frac{\theta}{2} H}
$$


where

$$
\begin{aligned}
E & =-\frac{1}{x} L_{\ell} j_{\ell}(x)+j_{\ell}^{\prime}(x), \\
F & =\frac{1}{x} j_{\ell}^{\prime}(x)+\frac{1}{x^{2}} L_{\ell} j_{\ell}(x)-\frac{1}{x} L_{\ell} j_{\ell}^{\prime}(x)-j_{\ell}^{\prime \prime}(x), \\
G & =\frac{1}{x} L_{\ell} h_{\ell}^{(1)}(x)-h_{\ell}^{(1) \prime}(x), \\
H & =-\frac{1}{x} h_{\ell}^{(1) \prime}(x)-\frac{1}{x^{2}} L_{\ell} h_{\ell}^{(1)}(x)+\frac{1}{x} L_{\ell} h_{\ell}^{(1) \prime}(x)+h_{\ell}^{(1) \prime \prime}(x),
\end{aligned}
$$

where $j_{\ell}(x)=\frac{1}{2}\left[h_{\ell}^{(1)}(x)+h_{\ell}^{(2)}(x)\right]$ and for real arguments, the spherical Hankel function of the second kind is the complex conjugate of the first one.

In the limit of $\theta \rightarrow 0, \alpha_{\ell}$ reduces to its commutative counterpart 22

$$
\alpha_{\ell}=-2 \frac{L_{\ell} j_{\ell}(x)-x j_{\ell}^{\prime}(x)}{L_{\ell} h_{\ell}^{(1)}(x)-x h_{\ell}^{(1) \prime}(x)} .
$$

After some calculations one can show that

$$
1+\alpha_{\ell}=-\frac{I+\frac{\theta}{2} K}{L+\frac{\theta}{2} M}
$$

where

$$
\begin{aligned}
I & =\frac{1}{x} L_{\ell} h_{\ell}^{(2)}(x)-h_{\ell}^{(2) \prime}(x), \\
K & =-\frac{1}{x^{2}} L_{\ell} h_{\ell}^{(2)}(x)+\frac{1}{x} L_{\ell} h_{\ell}^{(2) \prime}(x)-\frac{1}{x} h_{\ell}^{(2) \prime}(x)+h_{\ell}^{(2) \prime \prime}(x), \\
L & =\frac{1}{x} L_{\ell} h_{\ell}^{(1)}(x)-h_{\ell}^{(1) \prime}(x), \\
M & =-\frac{1}{x^{2}} L_{\ell} h_{\ell}^{(1)}(x)+\frac{1}{x} L_{\ell} h_{\ell}^{(1) \prime}(x)-\frac{1}{x} h_{\ell}^{(1) \prime}(x)+h_{\ell}^{(1) \prime \prime}(x) .
\end{aligned}
$$

The numerator of $1+\alpha_{\ell}$ is complex conjugate of the domeminator, so that

$$
\left|1+\alpha_{\ell}\right|=1 \text {. }
$$

This is an important result which shows the conservation of the number of particles in elastic scattering in noncommutative spaces because the absolute squares of the amplitudes of ingoing and outgoing waves must be equal. This is the unitarity relation for the $\ell$ th partial wave in a noncommutative space.

When $\theta \rightarrow 0$ it reduces to

$$
1+\alpha_{\ell}=-\frac{L_{\ell} h_{\ell}^{(2)}(x)-x h_{\ell}^{(2) \prime}(x)}{L_{\ell} h_{\ell}^{(1)}(x)-x h_{\ell}^{(1) \prime}(x)}
$$

which again satisfies in Eq.(33)! $! 22$

Up to now, we have shown the unitarity to the first order in $\theta$. One can show it for general case and to all order in $\theta$, we have

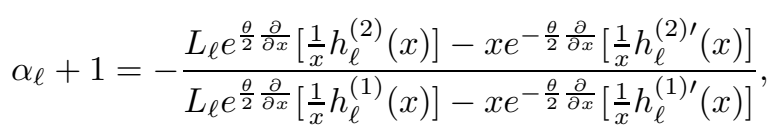


which satisfies in Eq.(33). To the zeroth and first order in $\theta$, Eq.(35) reduces to Eq.(34) and Eq.(28), respectively. The unitarity can be also shown without expanding in terms of Hankel functions. 4

Finally one can easily shown that the noncommutativity of space has no effect on the optical theorem and scattering amplitude of two identical particles, because

$$
\left(\frac{d \sigma}{d \Omega}\right)_{N C}=|f(\phi)|^{2}=f(\phi) \star f^{*}(\phi),
$$

where

$$
f(\phi)=\frac{1}{k} \sum_{\ell=0}^{\infty}(2 \ell+1) e^{i \delta_{\ell}(k)} \sin \delta_{\ell}(k) P_{\ell}(\cos \phi),
$$

but $\phi$ is the angle between the ingoing and outgoing particle momenta. This angle variable has nothing to do with $\mathrm{NC}$ variables introduced in Eq.(1) and therefore the star product in Eq.(36), reduces to ordinary produt. The same argument is true for the case of scattering of two identical particles, we have

$$
\begin{aligned}
\left(\frac{d \sigma}{d \Omega}\right)_{N C} & =|f(\phi)+f(\pi-\phi)|^{2} \\
& =f^{\star}(\phi) \star f(\phi)+f^{\star}(\pi-\phi) \star f(\pi-\phi)+f^{\star}(\phi) \star f(\pi-\phi) \\
& +f^{\star}(\pi-\phi) \star f(\phi),
\end{aligned}
$$

where again $\phi$ is the the angle between the ingoing and outgoing particle momenta (two particles), and therefore the noncommutativity of space has no effect on this amplitude. This means that again the star product reduces to ordinary product.

\section{Delta function potential}

Let us study scattering from delta function potential $V(\vec{r})=B \delta(\vec{r})$. The differential cross section in commutative case is isotropic

$$
\frac{d \sigma}{d \Omega}=\frac{\mu^{2} B^{2}}{4 \pi^{2} \hbar^{4}}
$$

where $\mu$ is the reduced mass. But we are not quite sure if we understand what a delta function potential in a NC setup would mean, because $\left[\hat{x}_{i}, \hat{x}_{j}\right]=i \theta_{i j}$ implies that we can not localize anything with infinite precision. Probably a good regulator for the delta function potential is a Gaussian function like $V(r)=\lambda \exp \left[-\left(\frac{r}{R}\right)^{2}\right]$, which generates delta function as we send $\theta$ to zero, if we take $R=\frac{1}{\lambda}=\theta$. So we consider the Gaussian function in a NC space. Using Eq.(8), one can calculate the corrections due to space noncommutativity on the amplitude. Then the cross section will have the following form

$$
\left(\frac{d \sigma}{d \Omega}\right)_{N C}=\frac{2 \pi m^{2} \lambda^{2}}{\hbar^{4}} R^{6} \exp \left(-\left(\frac{8 m E}{\hbar^{2}}\right) R^{2} \sin ^{2} \frac{\phi}{2}\right)\left[1+\frac{9 \theta^{2} p^{4}}{16 \hbar^{4}} \sin ^{2} \phi\right] .
$$


In conclusion some important aspects of scatterring in noncommutative spaces has been studied and these results are interesting to further understand the basic physics in noncommutative spaces.

\section{Acknowledgments}

I would like to thank P. Prešnajder (Comenius University, Slovakia) and M. M. Sheikh-Jabbari (Stanford University) for their careful reading of the manuscript and for their valuable comments.

\section{References}

1. M. Chaichian, M. M. Sheikh-Jabbari and A. Tureanu, Phys. Rev. Lett. 86, 2716 (2001).

2. M. Chaichian, A. Demichev, P. Prešnajder, M. M. Sheikh-Jabbari and A. Tureanu, Phys. Lett. B527, 149 (2002).

3. M. Chaichian, K. Nishijima and A. Tureanu, Phys. Lett. B568, 146 (2003).

4. M. Chaichian, C. Montonen and A. Tureanu, Phys. Lett. B566, 263 (2003).

5. P-M Ho and H-C Kao, Phys. Rev. Lett 88, 151602 (2001).

6. C. Zachos, Mod. Phys. Lett. A 19, 1483 (2004).

7. D. Kamani, Mod. Phys. Lett. A 182125 (2003).

8. A. Torrielli, Mod. Phys. Lett. A 182525 (2003).

9. R. Szabo, Int. J. Mod. Phys. Lett. A 191837 (2004).

10. I. V. Vancea, Phys. Lett. A 321, 155 (2004).

11. I. Avramidi, Phys. Lett. B576, 195 (2003).

12. M. Buric, V. Radovanovic, JHEP, 0402, 040 (2004).

13. J-Z Zhang, Phys. Lett. B584, 204 (2004).

14. J. Gamboa, F. Méndez, M. Loewe and J. C. Rojac, Mod. Phys. Lett. A 16, 2075 (2001).

15. B. Mirza, M. Zarei, Eur. Phys. J. C 32, 583 (2004).

16. S. A. Alavi, Chin. Phys. Lett. 20, 8 (2003).

17. S. A. Alavi, Physica Scripta, T67, 366 (2003).

18. S. A. Alavi, Physica Scripta (in press).

19. L. Mezincescu, hep-th/0007046

20. Y. Zunger, JHEP 0104, 039 (2001).

21. O. Espinosa and P. Gaete, hep-th/0206066

22. S. Flugge, Practical Quantum Mechanics, Springer-Verlag Berlin Heidelberg 1999. 